\title{
Successful anti-tachycardia pacing of a temporary implantable cardioverter-defibrillator used in the treatment of an arrhythmic storm
}

\author{
Anna Rydlewska ${ }^{1,2}$, Krzysztof Boczar ${ }^{1}$, Marcin Kuniewicz ${ }^{1,2}$, Barbara Małecka ${ }^{1,2}$, Jacek Lelakowski ${ }^{1,2}$ \\ 'Department of Electrocardiology, John Paul II Hospital, Krakow, Poland \\ ${ }^{2}$ Clinical Department of Electrocardiology, Institute of Cardiology, Jagiellonian University Collegium Medicum, \\ John Paul II Hospital, Krakow, Poland
}

A 70-year-old woman was admitted to hospital due to recurring symptomatic sustained ventricular tachycardias (VTs). She was diagnosed with ischaemic cardiomyopathy with low ejection fraction (20\%). She also had left bundle branch block and chronic heart failure in New York Heart Association class III. Blood electrolyte levels were normal. Coronary angiography showed a preserved effect of previous angioplasty. Clostridium difficile infection was identified. Increasing levels of C-reactive protein and procalcitonin were observed. Despite treatment with metoprolol ( $100 \mathrm{mg}$ per day) and amiodarone (1200 mg per day), VTs kept recurring; they had morphology of right bundle branch block with right axis deviation and cycle length of $240 \mathrm{~ms}$. Ablation was performed using an anatomical visualisation system (CARTO). Potential map of the left ventricle revealed a large scar region. During left ventricular mapping VTs of the same morphology were induced with programmed ventricular stimulation (PVS). Areas of low conduction were removed. After ablation of late potentials, PVS demonstrated non-induction of VT. No arrhythmias were observed directly after the ablation, but an iatrogenic third-degree atrioventricular block occurred. The patient required temporary stimulation with indications to implantation of a permanent cardiac resynchronisation therapy defibrillator (CRT-D). However, the implantation was contraindicated due to an active infection. Therefore, a temporary external implantable cardioverter-defibrillator (ICD) with dual-coil lead and a conventional ICD was implanted on the opposite side of the planned permanent implantation. The implantation parameters were: $\mathrm{R}$ wave $-14 \mathrm{mV}$, impedance $-537 \Omega$, threshold $-1.2 \mathrm{~V} / 0.5 \mathrm{~ms}$. Defibrillation threshold test was not performed due to the generally serious condition of the patient. Two detection zones were programmed. The therapy started with anti-tachycardia pacing (ATP) - three attempts of burst: 12 cycles of $88 \%$ in the VT zone (141 bpm), followed by cardioversions $(\mathrm{CVs})$, sending shocks between the two lead coils. Ventricular fibrillation zone $(214 \mathrm{bpm})$ therapy consisted only of CVs. The ICD can was placed on the chest wall and attached with adhesives. The patient was scheduled for further infection treatment. After nine days she had recurrent VTs which had similar morphology to those previously observed and were successfully treated by both ATPs and CVs from the temporary ICD (Fig. 1). Further hospitalisation was uneventful. After normalisation of the inflammatory parameters, a permanent CRT-D was implanted. At one and six months of follow-up the patient's condition improved with no arrhythmias or return of atrioventricular conduction. Treatment of an arrhythmic storm starts with the elimination of a potentially reversible cause, which can be established in about $66 \%$ of patients [1]. In this case, no such identifiable cause was found. In such situations treatment with antiarrhythmic medications, sedatives, and invasive methods is required. The OPTIC study showed that prevention of VT recurrence in patients with ICDs is effective with a combination of amiodarone and $\beta$-blockers [2]. In our patient, amiodarone with metoprolol did not prevent VT recurrence. The next step in the treatment is ablation, which not only stops electrical storm, but also improves long-term prognosis [3]. In our case VT returned shortly after ablation, probably due to the healing process after the procedure and an active infection. The infection was a reason against immediate implantation of a permanent CRT-D. We decided to use a bridge therapy with an external ICD with classic dual-coil lead, not only to ensure

A

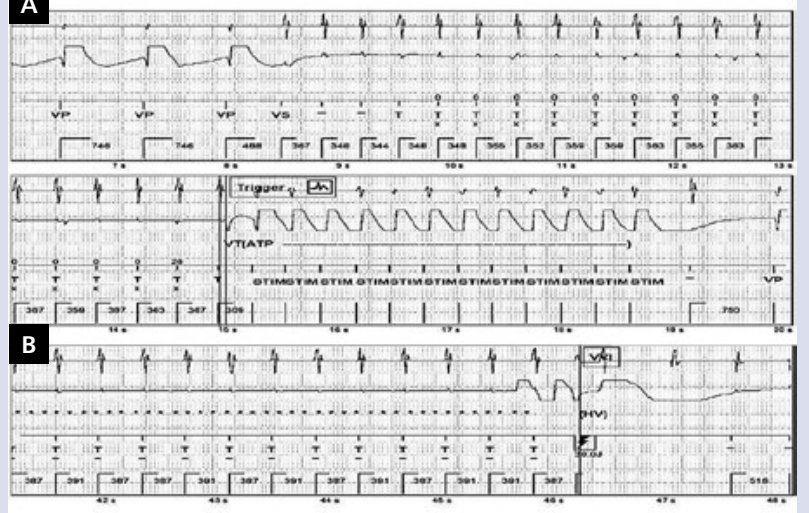

Figure 1. Ventricular tachycardias treated by anti-tachycardia pacing $(\mathbf{A})$ and cardioversions (B) by an external implantable cardioverter-defibrillator stimulation, but also to shorten the time of necessary treatment application in the case of VT recurrence. The dual-coil lead was implanted because the can was not placed under the skin, but externally. The defibrillation dipole was programmed from coil to coil in order to ensure appropriate defibrillation impedance and prevent skin burning during CV. It proved to be an effective treatment - both ATP and CV were successful. Programming of the VT zone was performed, although it was not line with the guidelines because previous VTs caused immediate decompensation of the patient.

\section{References}

1. Israel CW, Barold SS. Electrical storm in patients with an implanted defibrillator: a matter of definition. Ann Noninvasive Electrocardiol. 2007; 12(4): 375-382, doi: 10.1111/j.1542-474X.2007.00187.x, indexed in Pubmed: 17970963.

2. Connolly SJ, Dorian P, Roberts RS, et al. Comparison of beta-blockers, amiodarone plus beta-blockers, or sotalol for prevention of shocks from implantable cardioverter defibrillators: the OPTIC Study: a randomized trial. JAMA. 2006; 295(2): 165-171, doi: 10.1001/jama.295.2.165, indexed in Pubmed: 16403928.

3. Sagone A. Electrical storm: incidence, prognosis and therapy. J Atr Fibrillation. 2015; 8(4): 1150, doi: 10.4022/jafib.1150, indexed in Pubmed: 27957218.

\section{Address for correspondence:}

Anna Rydlewska, MD, PhD, Department of Electrocardiology, John Paul II Hospital, ul. Prądnicka 80, 31-202 Kraków, Poland, tel: +48 506351542 , e-mail: annarydlewska@op.pl

Conflict of interest: none declared

Kardiologia Polska Copyright (c) Polish Cardiac Society 2018 